\title{
Alternative splicing of bicistronic MOCS1 defines a novel mitochondrial protein maturation mechanism
}

Simon Julius Mayr ${ }^{1}$, Juliane Röper ${ }^{1}$ and Guenter Schwarz ${ }^{1,2,3, *}$

${ }^{1}$ Institute of Biochemistry, Department of Chemistry, University of Cologne, 50674 Cologne, Germany

${ }^{2}$ Center for Molecular Medicine Cologne, University of Cologne, 50931 Cologne, Germany

${ }^{3}$ Cluster of Excellence Cluster in Aging Research, University of Cologne, 50931 Cologne, Germany

* correspondence: gschwarz@uni-koeln.de

Running Title:

Mitochondrial MOCS1 protein maturation 


\section{Abstract}

Molybdenum cofactor biosynthesis is a conserved multistep pathway. The first step, the conversion of GTP to cyclic pyranopterin monophosphate (cPMP), requires bicsistronic MOCS1. Alternative splicing of MOCS1 in exons 1 and 9 produces four different $\mathrm{N}$-terminal and three different C-terminal products (type I-III). Type I splicing results in bicistronic transcripts with two open reading frames, of which only the first, MOCS1A, is translated, whereas type II/III splicing produces two-domain MOCS1AB proteins. Here, we report and characterize the mitochondrial translocation of alternatively spliced MOCS1 proteins. While MOCS1A requires exon 1a for mitochondrial translocation, MOCS1AB variants target to mitochondria via an internal motif overriding the $\mathrm{N}$-terminal targeting signal. Within mitochondria, MOCS1AB undergoes proteolytic cleavage resulting in mitochondrial matrix localization of the MOCS1B domain. In conclusion we found that MOCS1 produces two functional proteins, MOCS1A and MOCS1B, which follow different translocation routes before mitochondrial matrix import, where both proteins collectively catalyze cPMP biosynthesis. MOCS1 protein maturation provides a novel mechanism of alternative splicing ensuring the coordinated targeting of two functionally related mitochondrial proteins encoded by a single gene.

\section{Keywords}

alternative splicing / iron-sulfur cluster / mitochondrial translocation signal / molybdenum cofactor biosynthesis / radical SAM enzyme 


\section{Introduction}

The molybdenum cofactor (Moco) is found in all kingdoms of life forming the active center of molybdenum-containing enzymes, except nitrogenase. In mammals, there are four enzymes that depend on Moco: sulfite oxidase, xanthine oxidase, aldehyde oxidase and the mitochondrial amidoxime-reducing component (Schwarz et al., 2009). Moco is composed of an organic pterin moiety that binds molybdenum via a dithiolene group attached to a pyran ring, which is synthesized by a complex biosynthetic pathway. A mutational block in any step of Moco biosynthesis results in Moco deficiency (MoCD), a severe inborn error of metabolism characterized by rapidly progressing encephalopathy and early childhood death (Schwarz, 2005). In recent years, efficient treatment for Moco-deficient patients with a defect in the first step of Moco-biosynthesis has been established (Veldman et al., 2010, Schwahn et al., 2015).

Moco biosynthesis is divided into three major steps, starting with GTP followed by the synthesis of three intermediates: cyclic pyranopterin monophosphate (cPMP) (Santamaria-Araujo et al., 2004), molybdopterin or metal-binding pterin (MPT) (Schwarz, 2005) and adenylated MPT (Kuper et al., 2004). Due to the strict evolutionary conservation of the pathway, intermediates are identical in all kingdoms of life. The first and most complex reaction sequence is catalyzed by two proteins in bacteria (MoaA and MoaC) while in humans different translation products originating from different open reading frames (ORFs) of the MOCS1 gene (MOCS1A and MOCS1AB) are required for cPMP synthesis (Stallmeyer et al., 1999, Wuebbens et al., 2000, Hanzelmann et al., 2002).

E. coli MoaA and mammalian MOCS1A belong to the superfamily of radical Sadenosylmethionine (SAM) proteins with two highly oxygen-sensitive [4Fe-4S] clusters. The respective reaction mechanism has been studied for MoaA and involves reductive cleavage of SAM by the N-terminal [4Fe-4S] cluster (Hanzelmann et al., 2004). The resulting 5'deoxyadenosyl radical initiates the transformation of 5'-GTP, which is bound to a C-terminal [4Fe-4S] cluster, by abstracting the 3' proton from the ribose. Following a multi-step rearrangement reaction, $3,8{ }^{\prime} \mathrm{CH}_{2}$-GTP is released (Hover \& Yokoyama, 2015) and further 
processed by the second protein (E. coli MoaC, respectively human MOCS1AB) (Hanzelmann et al., 2002) resulting in pyrophosphate release and cyclic phosphate formation (Fig. 1A) (Wuebbens et al., 2000, Hover et al., 2015).

Interestingly, in the first two steps of Moco biosynthesis (cPMP and MPT synthesis) bicistronic transcripts (MOCS1 and MOCS2) have been reported (Reiss et al., 1998a, Stallmeyer et al., 1999), which is very unusual in human gene expression (Lu et al., 2013). Proteins involved in cPMP synthesis are encoded by the MOCS1 gene harboring 10 exons (Fig. 1B) leading to different alternatively spliced MOCS1 transcripts (Gray \& Nicholls, 2000), which are classified into three forms: Type I transcripts are bicistronic mRNAs with two non-overlapping ORFs, MOCS1A and MOCS1B (Reiss et al., 1998b), of which only the first ORF is translated yielding active MOCS1A. Type II and III transcripts are derived from two alternative splice sites within exon 9 , both resulting in the lack of the MOCS1A stop codon and an in-frame-fusion with the second ORF (MOCS1B) thus producing a monocistronic transcript. Consequently, MOCS1B is not expressed independently. Splice type II only lacks 15 nucleotides of exon 9, whereas in the type III variant the entire exon 9 is absent (Fig. 1C). The resulting MOCS1AB fusion proteins harbor an active MOCS1B domain and a catalytically inactive MOCS1A domain due to deletion of the last 2 residues harboring a conserved C-terminal double-glycine motif (Hanzelmann et al., 2002). Type I and type III variants present $41 \%$ and $55 \%$ of all MOCS1A transcripts, respectively, the remainder being type II transcripts (Arenas et al., 2009).

In addition to exon 9 alternative splicing, cDNA sequences with four different 5'-regions have been reported and were named after the first authors describing the respective variants. Two alternative ATG start codons are encoded either by exon 1a (present in LARIN and ARENAS variants) or exon 1b (present in REISS and GROSS variants). Due to the lack of an intronic 3' splice site in LARIN, exon 1a is spliced to exon 1d, while in ARENAS it is directly joined to exon 2 (subsequently referred to as MOCS1-ad and MOCS1-a). In a third REISS variant, exon $1 \mathrm{~b}$ is joined with exons $1 \mathrm{c}$ and $1 \mathrm{~d}$, while in GROSS, it is spliced together with exon $1 \mathrm{~d}$ (MOCS1bcd and MOCS1-bd)(Fig. 1C). MOCS1-ad and MOCS1-bcd transcripts were found in different 
organs, while expression levels of MOCS1-bd were found to be very low (Gross-Hardt \& Reiss, 2002).

MoCD-causing mutations have been found in MOCS1, MOCS2, MOCS3 and GPHN genes (Reiss et al., 2001, Reiss \& Hahnewald, 2011, Huijmans et al., 2017). Compared to their bacterial orthologues, human MOCS1 proteins exhibit N-terminal extensions of up to 56 residues depending on the length of the $\mathrm{N}$-terminal splicing product of MOCS1 exon 1 . In contrast, in plants two genes (Cnx2 and $C n \times 3)$ encode for proteins required for cPMP synthesis, both of which are characterized by extension of up to 112 residues encoding for classical N-terminal mitochondrial targeting signals (Teschner et al., 2010). Therefore, we proposed that MOCS1 proteins also localize to mitochondria and asked the question how alternative splicing of MOCS1 transcripts controls the function and localization of MOCS1 proteins.

We found that in the bicistronic MOCS1 transcripts (MOCS1A) exon 1 splicing results in translocation to the mitochondrial matrix when exon $1 \mathrm{a}$ is translated, while exon $1 \mathrm{~b}$ variants remain cytosolic. In contrast, all monocistronic transcripts (MOCS1AB) produced proteins that were imported into mitochondria, regardless of their exon 1 composition. Additional submitochondrial localization studies of the MOCS1AB proteins revealed that only the proteolytic MOCS1B cleavage product was imported into the mitochondrial matrix, while full-length MOCS1AB could only be witnessed on the outer mitochondrial membrane. 


\section{Results}

\section{Localization of MOCS1A proteins}

Given the N-terminal extension of the MOCS1 proteins compared to the homologous bacterial MoaA protein, first the different $\mathrm{N}$-terminal splice variants were investigated concerning their cellular localization, knowing that $\mathrm{N}$-terminal extensions may be involved cellular translocation processes. Based on the published sequences for MOCS1-a (Arenas et al., 2009), MOCS1ad (AF034374)(Reiss \& Hahnewald, 2011), MOCS1-bd (Gross-Hardt \& Reiss, 2002) and MOCS1-bcd (Reiss et al., 1998a) variants, the four exon 1 type I splice variants were created by fusion PCR and inserted into pEGFP-N1 and expressed in COS7 cells as EGFP fusion proteins. While the MOCS1A-ad-EGFP protein colocalized with the mitochondrial marker (Mitotracker)(Fig. 2A), a diffuse cytosolic distribution was observed for MOCS1A-bcd-EGFP (Fig. 2B), suggesting that exon 1a facilitates mitochondrial import, given that exon 1d and exons 2-9 are shared between MOCS1A-ad and MOCS1A-bcd. In accordance no difference in cellular localization between MOCS1A-a and MOCS1A-ad, as well as MOCS1A-bd and MOCS1A-bcd, could be observed (Fig. EV1).

To probe the role of exon 1a in mitochondrial targeting of MOCS1A, exon 1a was expressed as an EGFP fusion protein in COS7 cells resulting again in colocalization with the mitochondrial marker (Fig. 2C). Subsequent in silico analysis of the MOCS1A-ad sequence for mitochondrial translocation signals (MitoProtII)(Claros \& Vincens, 1996) revealed a high mitochondrial import probability (99.94\%) and a cleavage site after 22 residues (Fig. 3A). Subsequent expression of a fusion protein consisting of the $\mathrm{N}$-terminal 22 residues and EGFP indeed resulted in colocalization of EGFP and the mitochondrial marker (Fig. 2D), confirming the presence of a classical N-terminal import signal in exon 1a. In silico analysis of the MOCS1A-ad sequence using heliquest (Gautier et al., 2008) revealed the formation of an amphipathic helix within the N-terminal 22 residue between Arg4 and Cys21 (Fig. 3A).

MOCS1A proteins harbor two [4Fe4S], of which the N-terminal cluster is coordinated by two cysteines encoded by exon 1d. Expression of all four MOCS1A isoforms yielded in comparable 
activities of all splice variants except MOCS1A-a, which showed strongly reduced activity as expected due to the absence of exon 1d (Fig. EV2). This finding underlines the importance of fully coordinated clusters pointing towards an import into the mitochondrial matrix representing a possible compartment of iron-sulfer cluster proteins, besides the cytosol and the nucleus (Ciofi-Baffoni et al., 2018). In order to probe for matrix import of MOCS1A-ad, we expressed the MOCS1A-ad-EGFP fusion protein in HEK293 cells and enriched mitochondria after two days of culture. Enriched mitochondria were resuspended in two different buffers either keeping the mitochondria stable or inducing hypotonic swelling and thereby disrupting the outer mitochondrial membrane. Addition of proteinase $\mathrm{K}$ to one aliquot of each condition revealed that proteinase $\mathrm{K}$ was not able to digest MOCS1A-ad in both intact and swollen mitochondria (Fig. 3B), indicating mitochondrial matrix localization, as confirmed by the efficient digestion of SMAC protein (IMS loading control) in the proteinase $\mathrm{K}$ treated swollen mitochondria sample, but not of the GOT2 protein (matrix loading control). As a result, MOCS1A was found to be translocated to the mitochondrial matrix via a classical mitochondrial targeting signal located in exon 1 a.

\section{Localization of MOCS1AB proteins}

Following the results obtained for the MOCS1A proteins, we next investigated the cellular localization of MOCS1AB proteins (splice type III, encoded by exons 1-8 and exon 10). Similar to the experimental design for MOCS1A proteins, we expressed MOCS1AB-ad-EGFP and MOCS1AB-bcd-EGFP in COS7 cells. As expected, MOCS1AB-ad-EGFP colocalized with the mitochondrial marker (Fig. 4A), however, when we expressed the MOCS1AB-bcd-EGFP fusion protein we again observed mitochondrial localization (Fig. 4B), even though exon 1a encoded residues were not present. Testing splice type II variants resulted in mitochondrial localization independent of exon 1 composition as well (Fig. EV3). Since the exons 1-8 are shared between the type I (MOCS1A) and type III (MOCS1AB) splice variants our finding suggested the presence of an additional translocation signal encoded by exon 10 . 
Alignment of MOCS1AB with the homologous bacterial proteins MoaA and MoaC revealed a polypeptide sequence of 95 non-conserved residues located between the MOCS1A and MOCS1B domain, encoded by the 5' sequence of exon 10 (Fig. 4C). Such a sequence outside the functional domain might indicate an internal translocation signal, which was indeed confirmed by a stepwise TargetP in silico sequence analysis (Emanuelsson et al., 2000, Backes et al., 2018). Furthermore, subsequent heliquest sequence analysis showed the presence of another amphipathic helix resulting in a hydrophobic moment at position 395 (Gautier et al., 2008)(Fig. 5A). To probe this bioinformatic approach, full-length exon 10 (representing MOCS1B) and a deletion of the 5' 285 bases of exon 10 (representing MOCS1B 1 1-95 residues) were expressed in HEK293 cells using a pCDNA3.1 vector. To ensure that neither an $\mathrm{N}$-terminal nor a C-terminal translocation signal would be disturbed, no tags were fused to the construct, but an antibody was raised against recombinant MOCS1B $\Delta 1$ 95. HEK293 cells were harvested after two days of protein expression and fractionated into cytosolic (mitochondria free) and non-cytosolic fraction (containing mitochondria). Comparative western blot analysis of the obtained fractions revealed a strong enrichment of MOCS1B to the non-cytosolic fraction while the majority of MOCS1B $\Delta 1-95$ was retained in cytosolic fraction confirming the presence of an internal translocation signal at the $\mathrm{N}$-terminus of MOCS1B (Fig. 5B), which was in accordance with the loading controls VDAC (mitochondria) and gephyrin (cytosol). The cellular distribution of both MOCS1B variants was subsequently confirmed by transfecting COS7 cells with plasmids expressing both MOCS1B and MOCS1B $\triangle 1-95$ as EGFP fusion proteins. While the MOCS1B-EGFP fusion protein again localized to mitochondria (Fig. 5C), we found that the MOCS1B $\Delta 1-95-E G F P$ fusion showed a cytosolic distribution of the protein (Fig. 5D), demonstrating indeed the presence of an additional translocation signal in the exon 10 encoded linker region connecting the MOCS1Aand MOCS1B-domains.

\section{Sub-mitochondrial localization of MOCS1AB}


Considering that MOCS1A proteins are either cytosolic or mitochondrial matrix proteins, we next investigated the sub-mitochondrial localization of MOCS1AB proteins and whether this is influenced by their exon 1 composition. Therefore we first compared the mitochondrial distribution of MOCS1A-ad and MOCS1AB-bcd by expressing both proteins as N-terminal fusions to ratiometric pHluorin (Miesenbock et al., 1998) in HEK293 cells.

First we calibrated the ratiometric pHluorin protein to different $\mathrm{pH}$ values. Cells were harvested, fractionated and finally the non-cytosolic fractions were resuspended in buffers of different known $\mathrm{pH}$-values and disrupted resulting in excitation spectra differing in their excitation maxima at $388 \mathrm{~nm}$ and $456 \mathrm{~nm}$ in a ratiometric manner (Fig. 6A). As a result, the excitation peak at $388 \mathrm{~nm}$ was rising with increasing $\mathrm{pH}$, while the signal at $456 \mathrm{~nm}$ was decreasing accordingly. The ratio of the excitation bands $(388 \mathrm{~nm} / 456 \mathrm{~nm})$ was than related to the respective $\mathrm{pH}$-values and a sigmoidal fit curve was applied (Fig. 6B) allowing the calculation of an unknown $\mathrm{pH}$-value from determined excitation ratios.

Next, both MOCS1A-ad-pHluorin and MOCS1AB-bcd-pHluorin were expressed in HEK293 cells, harvested and fractionated. The non-cytosolic fraction was again resuspended in a buffer of known $\mathrm{pH}$ (7.4), but was left intact. Measurement of the intact mitochondria yielded $\mathrm{pH}$ values of $7.78 \pm 0.02$ for MOCS1A-ad and $7.90 \pm 0.03$ for MOCS1AB-bcd indicating matrix localization for both proteins when compared to the mitochondrial matrix protein GOT2 $(7.86 \pm 0.05)$. Following the measurement of the intact mitochondria, samples were disrupted and re-measured resulting in the expected pH-values of $7.41 \pm 0.05$ and $7.28 \pm 0.02$ for MOCS1A-ad and MOCS1AB-bcd resembling the $\mathrm{pH}$ of the used buffer and differing from the $\mathrm{pH}$ of the intact mitochondria significantly (Fig. 6C).

In addition to the ratiometric pHluorin studies, localization of MOCS1A-ad and MOCS1AB-bcd protein to the mitochondrial matrix strongly suggested matrix localization of MOCS1AB-ad as well. To confirm this proposal, MOCS1AB-ad was expressed in HEK293 cells without any tag using the pCDNA3.1 vector. Following enrichment of mitochondria the aforementioned proteinase $\mathrm{K}$ treatment was performed. Surprisingly MOCS1AB could not be detected by the 
MOCS1B antibody in either sample that was exposed to proteinase $\mathrm{K}$, but it was present in both control samples (Fig. 6D), suggesting that MOCS1AB is localized to the outer side of the outer mitochondrial membrane. The experiment was also repeated using EGFP fused MOCS1AB-bcd (Fig. EV4), with a similar result indicating that neither the EGFP nor the pHluorin tag, nor the exon 1 composition alters the translocation of MOCS1AB proteins. This finding was in contrast to the obtained results of the pHluorin measurements.

In addition to the bands observed for the full-length MOCS1AB proteins, the MOCS1B antibody also detected a band at approximately $20 \mathrm{kDa}$ in both proteinase $\mathrm{K}$ treated samples as well as untreated samples, but not in a control sample of disrupted mitochondria treated with proteinase $\mathrm{K}$ (Fig. 6D). A comparable $20 \mathrm{kDa}$ band was also detected when we used Cterminal-tag antibody (Fig. EV4). These findings suggests that a MOCS1B protein lacking the entire N-terminal MOCS1A domain, but not full-length MOCS1AB, was imported into the mitochondrial matrix. To provide further support for the exclusive localization of MOCS1B to the mitochondrial matrix, mitochondria were enriched from HEK293 cells overexpressing MOCS1AB-ad and exposed to an alkaline extraction of the mitochondrial membranes with the aim to separate the inner and outer mitochondrial membranes from the soluble fractions consisting of the intermembrane space and the mitochondrial matrix. Subsequent western blot analysis revealed an exclusive localization of full-length MOCS1AB to mitochondrial membranes while the proteolytically processed MOCS1B protein was mainly found in the soluble fraction, which was in line with the respective loading controls for IMS (sulfite oxidase) and outer membrane (VDAC ) (Fig. 6E). Therefore the full-length MOCS1AB protein represents the apo-protein of MOCS1B, which is proteolytically cleaved during procession to the mitochondrial matrix $\mathrm{N}$-terminal to the MOCS1B domain, resulting in a mature soluble MOCS1B protein. 


\section{Discussion}

In this study we were able to dissect the impact of alternative splicing of both exon 1 and exon 9 of MOCS1 on the complex maturation path of MOCS1 proteins leading to the mitochondrial localization of MOCS1A and MOCS1B. The major impact of exon 9 splicing on MOCS1 proteins has been known for years (Gray \& Nicholls, 2000), defining the expression of two different types of proteins, MOCS1A or MOCS1AB, respectively. The contribution of exon 1 splicing was not understood, except that exon $1 \mathrm{~d}$ was found to be essential for catalytic activity as it contains two conserved cysteine residues required for the coordination of the $\mathrm{N}$-terminal [4Fe-4S] cluster. This cluster is crucial for catalytic function of MOCS1A as it is assumed to generate an adenosyl radical in the same way as MoaA in bacteria (Hover \& Yokoyama, 2015). This proposal is supported by complementation experiments of MoaA-deficient E. coli cells demonstrating a loss of catalytic activity in the MOCS1A-a splice variant (Fig. EV2).

In this study, we were able to show that exon 1a and exon $1 \mathrm{~b}$ determine the cellular localization of MOCS1 proteins. While exon 1a-encoded residues mediated translocation of MOCS1A to the mitochondrial matrix, MOCS1A proteins with an N-terminal extension encoded by exon $1 \mathrm{~b}$ remained cytosolic. The function of exon 1c remains unknown, however it should be considered that exons $1 \mathrm{~b}-\mathrm{d}$ are not separated by introns (Gross-Hardt \& Reiss, 2002), but rather represent 1 exon which may be spliced partially, similar to exon 9. In either case, we observed that exon 1c is neither essential for catalytic activity (Fig. EV2) nor for translocation processes (Fig. EV1).

Most surprisingly, we found that splicing of exon 9, which is known to produce either active MOCS1A or active MOCS1B protein, overrides the cytosolic translocation of the two discovered cytosolic splice variants, in respect to their $\mathrm{N}$-terminal extension. This is a result of a sequence encoded by exon 10 representing a 95 residue linker region separating the conserved MOCS1A and MOCS1B domains, which is found in all type II and type III splice variants of MOCS1. This linker is facilitating import into the mitochondrial matrix and contains a mitochondrial peptidase cleavage site as witnessed in our translocation studies, proteolysis 
experiments, and bioinformatically predicted (using the MOCS1B sequence) by Mitoprot (Claros \& Vincens, 1996) at position 437 (MOCS1AB splice variant III). The remaining MOCS1B protein (residue 438-620) has a calculated molecular mass of $19.2 \mathrm{kDa}$, exactly matching the observed size of the cleavage product. Consistently, the recombinantly expressed MOCS1B protein (missing the entire 95 residue linker region) was observed at 18.4 $\mathrm{kDa}$, while the cleavage product was slightly larger due to the deletion of only $70 \mathrm{~N}$-terminal residues (Fig. EV5). A translocation signal and cleavage site prediction using Mitofates (Fukasawa et al., 2015) displayed a mitochondrial processing peptidase (MPP) cleavage site at position 432 (numbering according to splice type III).

Our findings promote the idea that ancestral MOCS1A and MOCS1B genes, both encoding their separate localization signals, were fused in vertebrates resulting in an internalization of the MOCS1B translocation signal. As a consequence, we favor the hypothesis of a TOM/TIM23- (Kang et al., 2018) mediated import of MOCS1AB proteins. We furthermore found that exon 1 splicing does not impact MOCS1B procession as observed in partial proteolysis experiments of different MOCS1AB splice variants (Fig. EV4). This is remarkable in light of the competing nature of additional translocation signal present in MOCS1AB-a/ad variants, which in turn suggests a hierarchical import. The fate of the N-terminal MOCS1AB cleavage product consisting of MOCS1A (missing the catalytically essential C-terminal double glycine motif encoded by exon 9) remains unknown.

A very recently reported MoCD type A patient case demonstrated that the expression of the MOCS1AB fusion protein is not strictly required for the biosynthetic activity of MOCS1 proteins. In this patient low amounts of MOCS1B protein (residues 425 - 620 in splice type III) were able to produce approximately $1 \%$ of wildtype Moco levels leading to a mild patient phenotype (Mayr et al., 2018).

In humans, all four genes encoding for Moco-synthetic proteins represent gene fusions (MOCS1, MOCS2, MOCS3 and GPHN). While MOCS2 encodes for a unique bicistronic mRNA with overlapping reading frames leading to the orchestrated expression of MOCS2A and 
MOCS2B proteins by a leaky scanning mechanism resulting in the assembly of the heterotetrameric MPT-synthase complex (Reiss et al., 1999), MOCS3 and GPHN encode for fusion proteins. MOCS3 is responsible for the reactivation of MOCS2A and harbors beside the NifS-like sulfurtransferase domain an additional rhodanese-like domain not present in prokaryotes (Matthies et al., 2004). Gephyrin combines two catalytic activities that are represented by two different Moco-synthetic proteins (MogA and MoeA) (Belaidi \& Schwarz, 2013). Orchestrated expression of proteins catalyzing subsequent reaction steps results in the formation of heterooligomers or fusion proteins, which ensures equal expression levels of cooperating proteins (MOCS2) or catalytic domains (MOCS3, gephyrin). MOCS1 represent an example that goes beyond the functional interaction by ensuring orchestrated maturation of MOCS1A and MOCS1B.

In aggregate, biosynthesis of Moco is an evolutionary ancient pathway with each of its reactions steps representing a complex chemical transformation. In particular the reaction mechanism of cPMP formation represents a radical based rearrangement reaction being entirely conserved in all kingdoms of life (Schwarz et al., 2009). Even though the underlying chemistry is highly conserved, the structures of the involved genes differ greatly. Tracking back the evolutionary event of the MOCS1 gene fusion by aligning MoaA and MoaC in selected species revealed that the gene fusion was not just gained in ophisthokonta, but is a shared feature of the unikonta, given that the amoeba Dictyostelium discoideum harbors a fused MOCS1 orthologue. Therefore the gene fusion event can be traced back to the unikonta/bikonta junction. Besides the above discussed functional benefits of gene fusions in Moco biosynthesis, in the case of MOCS3 and GPHN gene fusion served as evolutionary root for novel functions in higher eukaryotes (Fritschy et al., 2008, Judes et al., 2015).

In conclusion, in this study we were able to demonstrate that MOCS1 proteins target to the mitochondrial matrix. We revealed the effects of alternative splicing on MOCS1 proteins allowing a first comprehensive understanding of the interplay of alternative splicing, cellular translocation and proteolysis mediated maturation of MOCS1 proteins. 


\section{Material and methods}

\section{Materials, plasmids and bacterial strains}

Oligonucleotides for PCR and sequencing were purchased from Sigma. Restriction enzymes required were purchased from Fermentas. The T5 RNA polymerase-based bacterial pQE-80L expression vector was used for expression of MOCS1 splice variants in E. coli and purchased from Qiagen. Mammalian expression vectors used for localization studies in COS7 cells and expression in HEK293 cells were pEGPF-N1 and ppHluorin-N1 (derived from pEGFP-N1) and pcDNA3.1myc/His-A, respectively, were from Invitrogen. Bacterial strains $E$. coli wild type MC4100 (araD139 $\Delta$ (argF-lac)U169 rpsL150 relA1 flbB3501 deoC1 ptsF25 rbsR) and moaA ${ }^{-}$ and moaC- mutant strains ( $F$ - thr, leu his pro arg thi ade gal lacY malE xyl ara $m t /$ str $\operatorname{Tr} \lambda r$ ) were used for complementation studies.

\section{Cloning of MOCS1 splice variants}

Constructs were derived from pRitalX (kindly provided by J. Reiss, Goettingen) (Kugler et al., 2007) while exon 1a was synthesized in vitro (Genescript). Based on the published gene sequences (Reiss et al., 1998b, Gross-Hardt \& Reiss, 2002, Arenas et al., 2009, Reiss \& Hahnewald, 2011) the different splice variants were obtained by two-step PCR reactions fusing the respective exons. The identities of all generated constructs were confirmed by sequencing (GATC-Biotech/Eurofins Genomics).

\section{Transfection of COS7 and HEK293 cells and transient protein expression}

COS7 and human embryonic kidney (HEK293) cells were grown in Dulbecco modified essential medium supplemented with $10 \%$ fetal calf serum and L-glutamine. For transfection, cells were seeded into 12 -well plates $\left(40,000\right.$ cells/well) or $10 \mathrm{~cm}$ dishes $\left(4.5 \times 10^{6}\right.$ cells $\left./ \mathrm{dish}\right)$, transfected with Fugene (Promega) or polyethylenimine (PEI, Sigma), respectively, according to manufactures instructions. Transfected HEK293 cells were harvested and prepared for further analysis as described. 


\section{Fluorescence and co-localization analysis}

COS7 cells were grown on collagen-coated cover slips, transfected with the respective transgene and cultured for $24 \mathrm{~h}$. Cells were washed with PBS and mitochondria were stained with Mitotracker ${ }^{\circledR}$ Red CMXRos according to the manufacturer's protocol. Following Mitotracker ${ }^{\circledR}$ Red staining, cells were washed twice with PBS and fixed with $4 \%$ paraformaldehyde. Coverslips were mounted on microscope slides using Mowiol (Calbiochem) with 1-4-Diazabicyclo(2,2,2)-octan (DABCO, Merck) and dried at $37{ }^{\circ} \mathrm{C}$ over night. GFP and Mitotracker ${ }^{\circledR}$ Red fluorescence was visualized by confocal laser-scanning microscopy with TIEclipse (Nikon).

\section{Enrichment and sub-fractionation of mitochondria from transfected HEK293 cells}

Mitochondria were enriched from HEK293 cells expressing the respective MOCS1 splice variants using a modified previously described method (Mattiazzi et al., 2002). All fractions were sonicated for 30 seconds at $10 \%$ amplitude using a digital sonifier (Branson, Model 250D) and centrifuged at $20000 \times \mathrm{g}$ for $5 \mathrm{~min}$ at $4^{\circ} \mathrm{C}$ before determination of protein concentration (Roti@-Quant; Roth, Germany) and supplementation with SDS loading buffer to remove protein aggregates. For partial proteolysis experiments enriched mitochondria were separated in 100 $\mu \mathrm{g}$ pellets, which were subsequently resuspended in $100 \mu \mathrm{l} 10 \mathrm{mM}$ HEPES buffer $\mathrm{pH} 7.6$ containing $1 \mathrm{mM} \mathrm{CaCl}_{2}$. For the samples of intact mitochondria the buffer additionally contained $220 \mathrm{mM}$ mannitol and $70 \mathrm{mM}$ sucrose. After $5 \mathrm{~min}$ incubation on ice the control sample was sonicated for 30 seconds at $10 \%$ amplitude and $2 \mu \mathrm{l}$ of a $5 \mathrm{mg} / \mathrm{ml}$ proteinase K (Roth, Germany) solution were added to the respective samples (swollen and swollen + sonicated). After additional 10 min incubation on ice PMSF (final concentration $1 \mathrm{mM}$ ) was added and proteins were participated using trichloroacetic acid (final concentration 5\%) for 10 min on ice.

Membrane and soluble mitochondrial proteins were fractionated by alkaline extraction. Therefore enriched mitochondria were resuspended in $0.1 \mathrm{M} \mathrm{Na}_{2} \mathrm{CO}_{3}, \mathrm{pH} 11.5$. After 30 min incubation on ice, mitochondria were centrifuged at $70000 \times \mathrm{g}$ for $1 \mathrm{~h}$ at $4{ }^{\circ} \mathrm{C}$. Resulting pellets 
containing the mitochondrial membranes were washed with $\mathrm{H}_{2} \mathrm{O}$ and resuspended in SDS loading buffer. The supernatants were concentrated using a $3 \mathrm{kDa}$ cutoff protein concentrator and supplemented with SDS loading buffer.

\section{Western blot analysis}

Localization of proteins of fractionated HEK293 cells and sub-fractionated mitochondria was analyzed by Western blot following SDS-PAGE and subsequent semi-dry blot. For the detection of MOCS1 proteins the following antibodies were used in the respective dilutions: anti-MOCS1A (rabbit polyclonal, Abcam, ab176989, 1/200), anti-MOCS1B (rabbit polyclonal, Eurogentec, rabbit SY7796, 1/100), anti-GFP-tag (rabbit polyclonal, Invitrogen, A6455, 1/1000) and anti-MYC-tag (mouse monoclonal, cell supernatant 9E10, 1/5). To ensure successful fractionation and sub-fractionation the following antibodies were used as controls: anti-GOT2 (Sigma, rabbit polyclonal, SAB2100-950, 1/500), anti-gephyrin (mouse monoclonal, cell supernatant 3B11, 1/20), anti-SMAC/Diablo (rabbit polyclonal, Abcam, ab8114, 1/2000), antisulfite oxidase (mouse monoclonal, Abcam, ab57852, 1/1000) and anti-VDAC1/Porin (rabbit polyclonal, Abcam, ab15895, 1/1000). All antibodies were diluted in TBST containing $2 \%$ dried non-fat milk powder.

\section{MOCS1B antibody}

The MOCS1B domain (encoded by exon $10 \Delta 1-285$ ) was expressed in E. coli BL21 as Nterminally His-tagged protein using a pQE80-L vector. Purification was achieved using Ni-NTA resign. The protein was eluted using a potassium phosphate $\mathrm{pH} 8.0$ buffer containing $\mathrm{NaCl}$ and imidazole $(250 \mathrm{mM})$. Subsequently the buffer was exchanged to a $20 \mathrm{mM}$ Tris- $\mathrm{HCl}$ buffer pH 7.8 containing $200 \mathrm{mM} \mathrm{NaCl}$ using a PD10-column (GE Healthcare) and diluted to 10 $\mathrm{mg} / \mathrm{ml}$. Purity of the protein was confirmed by a $12 \%$ SDS-PAGE using Coomassie-staining. The antibody was subsequently produced using the acquired protein as antigen (Eurogentech) and tested against different antigen concentrations $(0.2-20 \mathrm{ng})$, as well as recombinantly expressed MOCS1AB from HEK293 cell extract. 


\section{Determination of intra-mitochondrial $\mathrm{pH}$-values}

Mitochondria were enriched from HEK293 cells overexpressing the desired proteins as pHluorin fusions. For the determination of the pHluorin pKa-curves pellets of enriched mitochondria were resuspended in water $(2 \mathrm{mg} / \mathrm{ml})$ and sonicated for $30 \mathrm{~s}$ at $10 \%$ amplitude. Subsequently, $100 \mu$ l extract were mixed with $100 \mu \mathrm{l} 2$ x buffer (50 mM MES or Tris-HCl buffer $\mathrm{pH}$ 5.5-8.3, $0.3 \mathrm{M} \mathrm{NaCl}$ ) and excitation spectra were recorded from $350 \mathrm{~nm}$ to $500 \mathrm{~nm}$ at an emission wavelength of $510 \mathrm{~nm}$ at room temperature using a microplate reader (Tecan InfiniteM200). To measure the $\mathrm{pH}$-values of pHluorin fusions in intact mitochondria, pellets of enriched mitochondria were resuspended in buffer $\left(\mathrm{pH} 7.4\right.$ at $37^{\circ} \mathrm{C} ; 1 \mathrm{mg}$ enriched mitochondria/ml) and fluorescence was immediately determined as described above, but at $37^{\circ} \mathrm{C}$. Subsequently, each sample was sonicated for $30 \mathrm{~s}(10 \%$ amplitude) and remeasured.

\section{Determination of cPMP and MPT content}

cPMP was detected as Compound Z in crude cell extracts as described (Hanzelmann et al., 2002). In brief, protein extracts ( $0.5 \mathrm{mg}$ total protein) were oxidized, purified by quarternary aminoethyl column chromatography and HPLC analysis (Agilent Technologies) was performed using a C18-reversed phase column (Waters). Elution from quarternary aminoethyl columns was performed with $50 \mathrm{mM} \mathrm{HCl}$. cPMP concentrations were expressed in picomoles of compound Z per milligram of total protein.

\section{Statistics}

If not stated otherwise, all numerical data were reported as mean \pm standard deviation. All measurements were performed at least in triplicates. Statistical analysis (unpaired t-test) was performed with SigmaPlot (Systat Software Inc). Significance levels are indicated in figures as ${ }^{*} p<0.05,{ }^{* *} p<0.01,{ }^{* *} p<0.001$. 


\section{Acknowledgments}

Technical assistance by Monika Laurien (University of Cologne, Germany) is gratefully acknowledged. We thank Dr. Jochen Reiss (University of Goettingen) for providing the plasmid pRitalX and Prof. Reinhart Krämer for providing the pHluorin sequence. We thank Prof. Johannes Hermann and Prof. Timo Mühlhaus for the iMTS-L propensity analysis. We gratefully acknowledge funding by the Deutsche Forschungsgemeinschaft within the SPP1927 priority program.

\section{Author Contributions}

S.J.M. was involved in cloning of plasmids and cell culture work. S.J.M conducted all western bolt experiments and pHluorin $\mathrm{pH}$ determinations, was involved in study design and wrote the paper. J.R. was involved in cloning of plasmids and cell culture work. J.R. performed all microscopy and complementation experiments and was involved in study design. G.S. was involved in study design and wrote the paper.

\section{Declaration of Interest}

The authors declare no competing interests. 


\section{References}

Arenas M, Fairbanks LD, Vijayakumar K, Carr L, Escuredo E, Marinaki AM (2009) An unusual genetic variant in the MOCS1 gene leads to complete missplicing of an alternatively spliced exon in a patient with molybdenum cofactor deficiency. J Inherit Metab Dis 32: 560-9

Backes S, Hess S, Boos F, Woellhaf MW, Godel S, Jung M, Muhlhaus T, Herrmann JM (2018) Tom70 enhances mitochondrial preprotein import efficiency by binding to internal targeting sequences. J Cell Biol 217: 1369-1382

Belaidi AA, Schwarz G (2013) Metal insertion into the molybdenum cofactor: product-substrate channelling demonstrates the functional origin of domain fusion in gephyrin. Biochem J 450: 149-57 Ciofi-Baffoni S, Nasta V, Banci L (2018) Protein networks in the maturation of human iron-sulfur proteins. Metallomics 10: 49-72

Claros MG, Vincens P (1996) Computational method to predict mitochondrially imported proteins and their targeting sequences. Eur J Biochem 241: 779-86

Emanuelsson O, Nielsen H, Brunak S, von Heijne G (2000) Predicting subcellular localization of proteins based on their N-terminal amino acid sequence. J Mol Biol 300: 1005-16

Fritschy JM, Harvey RJ, Schwarz G (2008) Gephyrin: where do we stand, where do we go? Trends Neurosci 31: 257-64

Fukasawa Y, Tsuji J, Fu SC, Tomii K, Horton P, Imai K (2015) MitoFates: improved prediction of mitochondrial targeting sequences and their cleavage sites. Mol Cell Proteomics 14: 1113-26 Gautier R, Douguet D, Antonny B, Drin G (2008) HELIQUEST: a web server to screen sequences with specific alpha-helical properties. Bioinformatics 24: 2101-2

Gray TA, Nicholls RD (2000) Diverse splicing mechanisms fuse the evolutionarily conserved bicistronic MOCS1A and MOCS1B open reading frames. RNA 6: 928-36

Gross-Hardt S, Reiss J (2002) The bicistronic MOCS1 gene has alternative start codons on two mutually exclusive exons. Mol Genet Metab 76: 340-3

Hanzelmann P, Hernandez HL, Menzel C, Garcia-Serres R, Huynh BH, Johnson MK, Mendel RR, Schindelin $\mathrm{H}$ (2004) Characterization of MOCS1A, an oxygen-sensitive iron-sulfur protein involved in human molybdenum cofactor biosynthesis. J Biol Chem 279: 34721-32

Hanzelmann P, Schwarz G, Mendel RR (2002) Functionality of alternative splice forms of the first enzymes involved in human molybdenum cofactor biosynthesis. J Biol Chem 277: 18303-12 Hover BM, Tonthat NK, Schumacher MA, Yokoyama K (2015) Mechanism of pyranopterin ring formation in molybdenum cofactor biosynthesis. Proc Natl Acad Sci U S A 112: 6347-52

Hover BM, Yokoyama K (2015) C-Terminal glycine-gated radical initiation by GTP 3',8-cyclase in the molybdenum cofactor biosynthesis. J Am Chem Soc 137: 3352-9

Huijmans JGM, Schot R, de Klerk JBC, Williams M, de Coo RFM, Duran M, Verheijen FW, van Slegtenhorst M, Mancini GMS (2017) Molybdenum cofactor deficiency: Identification of a patient with homozygote mutation in the MOCS3 gene. Am J Med Genet A 173: 1601-1606

Judes A, Ebert F, Bar C, Thuring KL, Harrer A, Klassen R, Helm M, Stark MJ, Schaffrath R (2015) Urmylation and tRNA thiolation functions of ubiquitin-like Uba4.Urm1 systems are conserved from yeast to man. FEBS Lett 589: 904-9

Kang Y, Fielden LF, Stojanovski D (2018) Mitochondrial protein transport in health and disease. Semin Cell Dev Biol 76: 142-153

Kugler S, Hahnewald R, Garrido M, Reiss J (2007) Long-term rescue of a lethal inherited disease by adeno-associated virus-mediated gene transfer in a mouse model of molybdenum-cofactor deficiency. Am J Hum Genet 80: 291-7

Kuper J, Llamas A, Hecht HJ, Mendel RR, Schwarz G (2004) Structure of the molybdopterin-bound Cnx1G domain links molybdenum and copper metabolism. Nature 430: 803-6

Lu Y, Zhang Y, Hang X, Qu W, Lubec G, Chen C, Zhang C (2013) Genome-wide computational identification of bicistronic mRNA in humans. Amino Acids 44: 597-606 
Matthies A, Rajagopalan KV, Mendel RR, Leimkuhler S (2004) Evidence for the physiological role of a rhodanese-like protein for the biosynthesis of the molybdenum cofactor in humans. Proc Natl Acad Sci U S A 101: 5946-51

Mattiazzi M, D'Aurelio M, Gajewski CD, Martushova K, Kiaei M, Beal MF, Manfredi G (2002) Mutated human SOD1 causes dysfunction of oxidative phosphorylation in mitochondria of transgenic mice. J Biol Chem 277: 29626-33

Mayr SJ, Sass JO, Vry J, Kirschner J, Mader I, Hovener JB, Reiss J, Santamaria-Araujo JA, Schwarz G, Grunert SC (2018) A mild case of molybdenum cofactor deficiency defines an alternative route of MOCS1 protein maturation. J Inherit Metab Dis 41: 187-196

Miesenbock G, De Angelis DA, Rothman JE (1998) Visualizing secretion and synaptic transmission with $\mathrm{pH}$-sensitive green fluorescent proteins. Nature 394: 192-5

Reiss J, Christensen E, Kurlemann G, Zabot MT, Dorche C (1998a) Genomic structure and mutational spectrum of the bicistronic MOCS1 gene defective in molybdenum cofactor deficiency type A. Hum Genet 103: 639-44

Reiss J, Cohen N, Dorche C, Mandel H, Mendel RR, Stallmeyer B, Zabot MT, Dierks T (1998b) Mutations in a polycistronic nuclear gene associated with molybdenum cofactor deficiency. Nat Genet 20: 51-3

Reiss J, Dorche C, Stallmeyer B, Mendel RR, Cohen N, Zabot MT (1999) Human molybdopterin synthase gene: genomic structure and mutations in molybdenum cofactor deficiency type B. Am J Hum Genet 64: 706-11

Reiss J, Gross-Hardt S, Christensen E, Schmidt P, Mendel RR, Schwarz G (2001) A mutation in the gene for the neurotransmitter receptor-clustering protein gephyrin causes a novel form of molybdenum cofactor deficiency. Am J Hum Genet 68: 208-13

Reiss J, Hahnewald R (2011) Molybdenum cofactor deficiency: Mutations in GPHN, MOCS1, and MOCS2. Hum Mutat 32: 10-8

Santamaria-Araujo JA, Fischer B, Otte T, Nimtz M, Mendel RR, Wray V, Schwarz G (2004) The tetrahydropyranopterin structure of the sulfur-free and metal-free molybdenum cofactor precursor. J Biol Chem 279: 15994-9

Schwahn BC, Van Spronsen FJ, Belaidi AA, Bowhay S, Christodoulou J, Derks TG, Hennermann JB, Jameson E, Konig K, McGregor TL, Font-Montgomery E, Santamaria-Araujo JA, Santra S, Vaidya M, Vierzig A, Wassmer E, Weis I, Wong FY, Veldman A, Schwarz G (2015) Efficacy and safety of cyclic pyranopterin monophosphate substitution in severe molybdenum cofactor deficiency type $A$ : a prospective cohort study. Lancet 386: 1955-63

Schwarz G (2005) Molybdenum cofactor biosynthesis and deficiency. Cell Mol Life Sci 62: 2792-810 Schwarz G, Mendel RR, Ribbe MW (2009) Molybdenum cofactors, enzymes and pathways. Nature 460: 839-47

Stallmeyer B, Drugeon G, Reiss J, Haenni AL, Mendel RR (1999) Human molybdopterin synthase gene: identification of a bicistronic transcript with overlapping reading frames. Am J Hum Genet 64: 698705

Teschner J, Lachmann N, Schulze J, Geisler M, Selbach K, Santamaria-Araujo J, Balk J, Mendel RR, Bittner F (2010) A novel role for Arabidopsis mitochondrial ABC transporter ATM3 in molybdenum cofactor biosynthesis. Plant Cell 22: 468-80

Veldman A, Santamaria-Araujo JA, Sollazzo S, Pitt J, Gianello R, Yaplito-Lee J, Wong F, Ramsden CA, Reiss J, Cook I, Fairweather J, Schwarz G (2010) Successful treatment of molybdenum cofactor deficiency type A with cPMP. Pediatrics 125: e1249-54

Wuebbens MM, Liu MT, Rajagopalan K, Schindelin H (2000) Insights into molybdenum cofactor deficiency provided by the crystal structure of the molybdenum cofactor biosynthesis protein MoaC. Structure 8: 709-18 


\section{Figure legends}

Figure 1. Alternative splicing of the MOCS1 gene and gene products involved in cPMP synthesis. A) Biosynthesis of cPMP by the MOCS1 proteins in a two sub-step reaction. The MOCS1A protein converts GTP to the cyclic-dihydro-GTP intermediate, followed by the conversion of the intermediate to $\mathrm{CPMP}$ by MOCS1AB. B) Exon structure of the MOCS1 gene highlighting the four alternatively splice exon 1 cassettes (white boxes), the shared exons 2-8 (Black boxes), the alternatively spliced exon 9 (white box) encoding the C-terminal double glycine motif (GG) and stop codon $\left({ }^{*}\right)$, as well as the MOCS1B encoding exon 10 (black box). Introns are indicated by a black line but are not reflecting actual intron size. C) Alternative splicing of MOCS1 resulting in 12 different MOCS1 proteins. The splicing of exon 1 creates four alternative combinations (white boxes), while exon 9 splicing produces either the bicistronic type I variants when exon 9 (white box) is not spliced out leaving the double glycine motif and the stop codon in place. As a consequence active MOCS1A (exons 1-9), but not MOCS1B (exon 10) is translated. The monocistronic type II and III variants on do not contain the double glycine motif and the stop codon by having exon 9 removed either partially (type II) or entirely (type III), resulting in a MOCS1AB fusion protein representing active MOCS1B.

\section{Figure 2. Transient expression of MOCS1A splice variants and MOCS1 derived} constructs as GFP fusions in COS7 cells. COS7 cells were transfected with A) MOCS1Aad, B) MOCS1A-bcd, C) exon 1a and D) residues 1-22 encoded by exon 1a. Following $48 \mathrm{~h}$ of transient expression, mitochondria were stained with MitoTrackerßRed CMXRos and analyzed by confocal laser scanning microscopy. Overlay between the red and green (GFP) channel is shown in the yellow merge panel. Scale bars: $10 \mu \mathrm{m}$

Figure 3. Mitochondrial import of MOCS1A. A) Prediction of N-terminal amphipathic helix consisting of the residues 4 to 21 encoded by exon 1a (heliquest). B) Partial proteolysis experiment of mitochondria enriched from HEK293 cells overexpressing MOCS1A-ad as a GFP fusion protein. One of each fraction of intact and swollen mitochondria were treated with 
proteinase K. Subsequent $10 \%$-SDS-PAGE followed by western blot analysis was performed using anti-MOCS1, anti-SMAC and anti-GOT2 antibodies.

Figure 4. Identification of internal translocation signal of MOCS1AB proteins. $A, B$ ) Transient expression of MOCS1AB splice variants as GFP fusions in COS7 cells. COS7 cells were transfected with A) MOCS1AB-ad and B) MOCS1A-bcd. Following $48 \mathrm{~h}$ of transient expression, mitochondria were stained with MitoTracker®Red CMXRos and analyzed by confocal laser scanning microscopy. Overlay between the red and green (GFP) channel is shown in the yellow merge panel. C) Alignment of MOCS1AB (white box) with E. coli MoaA and MoaC (black boxes). Numbers indicate the positions of residues defining the $\mathrm{N}$-terminal extension (gray box) and the internal extension (red box).

Figure 5. Identification of exon 10 encoded MOCS1B translocation signal. A) TargetP and heliquest in silico analysis of the MOCS1AB amino acid sequence showing the C-terminal residues 300 to 600 . B) Cellular fractionation of HEK293 cells transiently expressing the exon 10 encoded either full-length MOCS1B protein or a truncated MOCS1B protein not containing the N-terminal 95 residues. Western blot analysis following $12 \%$ SDS-PAGE was performed using anti-MOCS1B, anti-gephyrin and anti-VDAC antibodies. C,D) Transient expression of C) exon 10 and D) exon $10 \Delta 1-285$ as GFP fusions in COS7 cells. Following $48 \mathrm{~h}$ of transient expression, mitochondria were stained with MitoTracker®Red CMXRos and analyzed by confocal laser scanning microscopy. Overlay between the red and green (GFP) channel is shown in the yellow merge panel.

Figure 6. Submitochondrial localization of MOCS1AB proteins. A-C) Excitation-scan measurements of enriched mitochondria from HEK293 cells transiently expressing MOCS1Aad, MOCS1AB-bcd or GOT2 as pHluorin fusions at $510 \mathrm{~nm}$ emission wavelength. A) MOCS1ad excitation spectra of disrupted mitochondria in buffer of different known $\mathrm{pH}$-values. B) Sigmoidal-fit calibration curve of pHluorin of the excitation maxima ratios $(388 \mathrm{~nm} / 456 \mathrm{~nm})$ versus the $\mathrm{pH}$-values. C) Fluorescence based $\mathrm{pH}$-determination of intact mitochondria (black bar) and disrupted mitochondria (grey bar) using the pHluorin calibration curve. D) Partial 
proteolysis experiment of mitochondria enriched from HEK293 cells overexpressing MOCS1AB-ad without tag. One of each fraction of intact and swollen mitochondria were treated with proteinase $\mathrm{K}$, as well as one additional sample of disrupted mitochondria. Subsequent $12 \%$-SDS-PAGE followed by western blot analysis was performed using antiMOCS1B, anti-SMAC and anti-GOT2 antibodies. E) Western blot analysis of sub-fractionated HEK293 cells. Mitochondrial membranes and soluble mitochondrial fractions were separated by alkaline extraction and subsequent ultra-centrifugation. Successful separation was demonstrated using anti-sulfite oxidase and anti-VDAC antibodies. Unprocessed MOCS1AB and mature MOCS1B were visualized using anti-MOCS1B antibody.

\section{Extended View Figure legends}

Extended View figure 1. Transient expression of MOCS1A and MOCS1AB (type III) splice variants as GFP fusions in COS7 cells. COS7 cells were transfected with MOCS1A-a (A), MOCS1A-bd (B), MOCS1AB-a (C) and MOCS1AB-bd (D). Following $48 \mathrm{~h}$ of transient expression, mitochondria were stained with MitoTracker®Red CMXRos and analyzed by confocal laser scanning microscopy. Overlay between the red and green (GFP) channel is shown in the yellow merge panel.

Extended View figure 2. Functional reconstitution of Moco-deficient E. coli mutants KB 5242 (moaA-)with the respective MOCS1 proteins. HPLC analysis of Compound Z (cPMP oxidation product) in crude cell extracts. Measurements were performed in triplicate and standard deviations of at least three independent experiments with triplicate measurements are indicated by the error bars. Statistical analysis using the t-test: ${ }^{* * *} p \leq 0.001,{ }^{* *} p \leq 0.01$ and ${ }^{*} p \leq 0.05$

Extended View figure 3. Transient expression of MOCS1AB (type II) splice variants as GFP fusions in COS7 cells. COS7 cells were transfected with MOCS1AB-a (A), MOCS1ABad (B), MOCS1AB-bd (C) and MOCS1AB-bcd (D). Following $48 \mathrm{~h}$ of transient expression, mitochondria were stained with MitoTracker®Red CMXRos and analyzed by confocal laser 
scanning microscopy. Overlay between the red and green (GFP) channel is shown in the yellow merge panel.

Extended View figure 4. Partial proteolysis experiment of mitochondria enriched from HEK293 cells. HEK293 cells overexpressing MOCS1AB-bcd-MYC (A) or MOCS1AB-ad-GFP (B) fusion proteins were fractionated and partial proteolysis was performed. One of each fraction of intact and swollen mitochondria were treated with proteinase $\mathrm{K}$, as well as one additional sample of disrupted mitochondria. Subsequent 12\%-SDS-PAGE followed by western blot analysis was performed using anti-SMAC and anti-GOT2 antibodies as loading controls. Tagged MOCS1AB proteins were detected using anti-MYC (A) or anti-GFP (B) antibodies.

Extended View figure 5. MOCS1B antigen and antibody. A) Coomassie-stained $12 \%$ SDSPAGE of recombinantly expressed, purified His-tagged MOCS1B domain (exon 10 missing 5' 285 bases) serving as MOCS1B antigen B) WB of $12 \%$ SDS-PAGE of $10 \mu \mathrm{g}$ enriched mitochondria from HEK293-cells overexpressing MOCS1AB and of purified MOCS1B antigen (0.2 ng - $2 \mathrm{ng})$. 


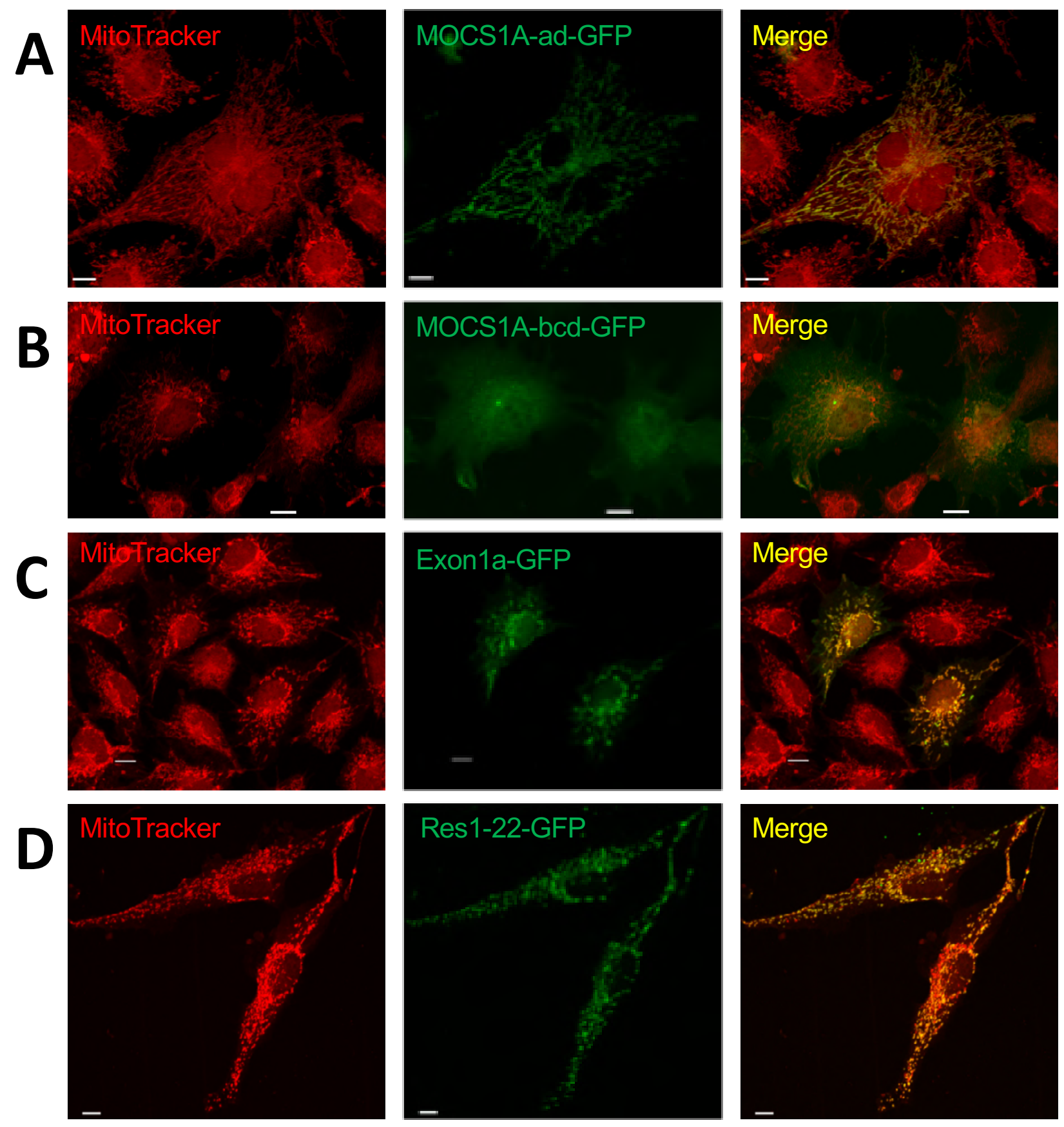

Fig 2

Mayr et al. 


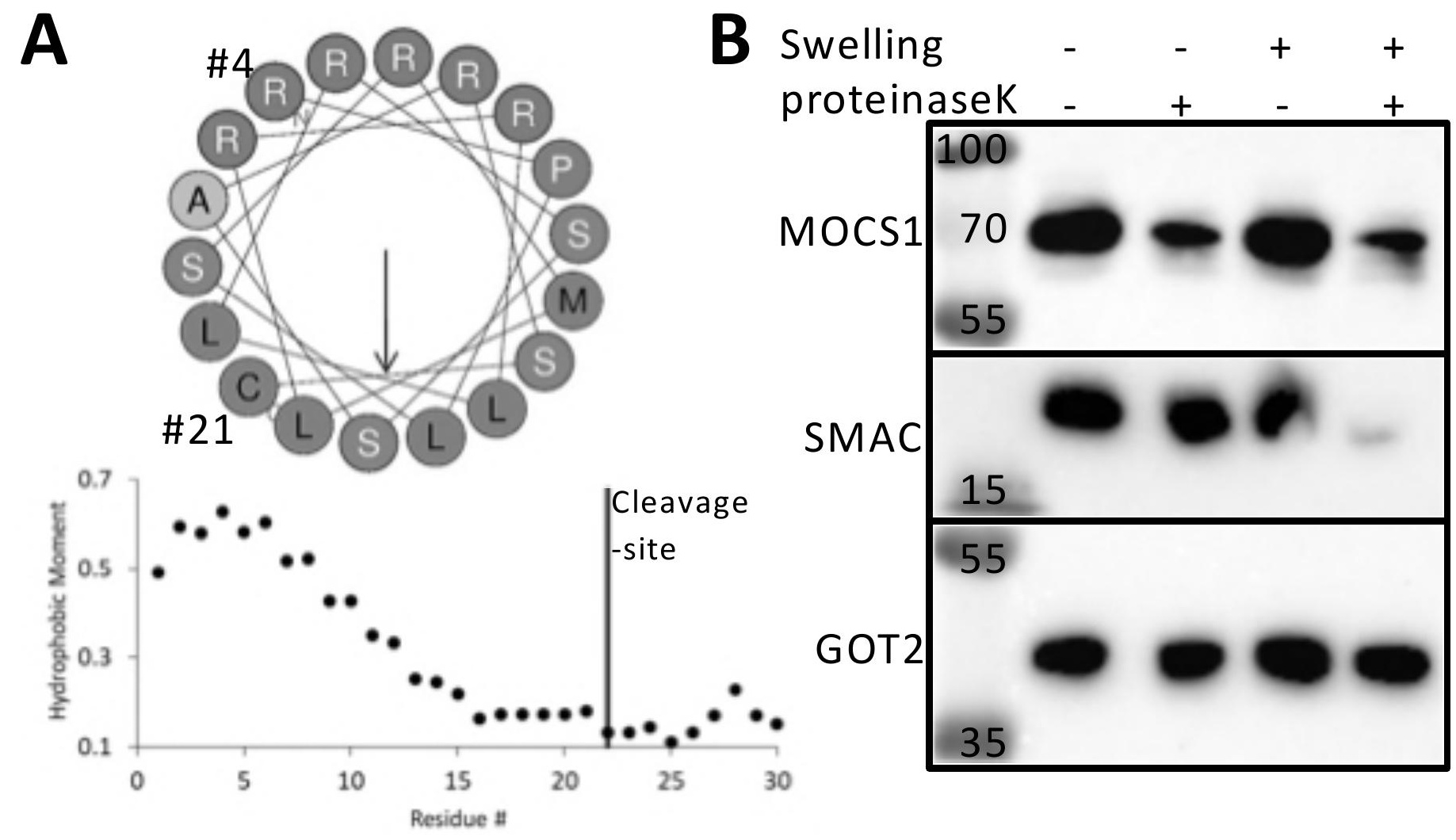

Fig 3

Mayr et al. 

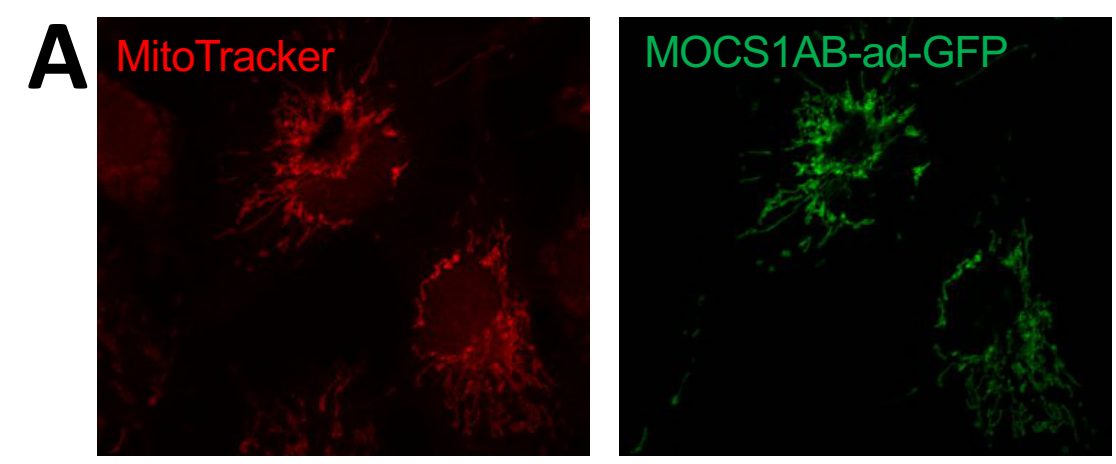

B
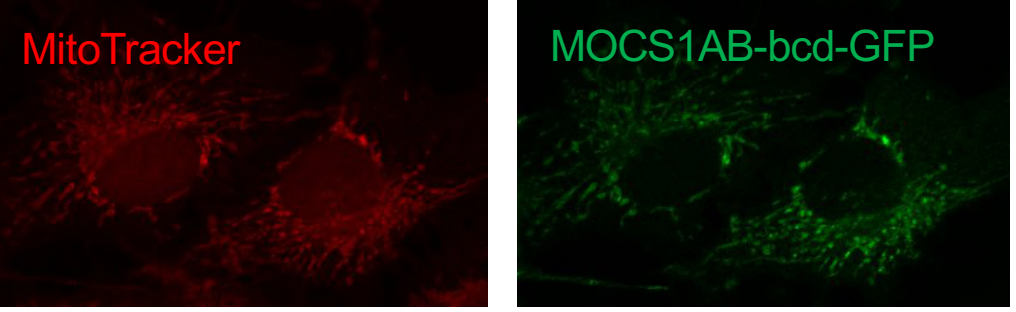

c

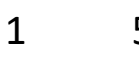

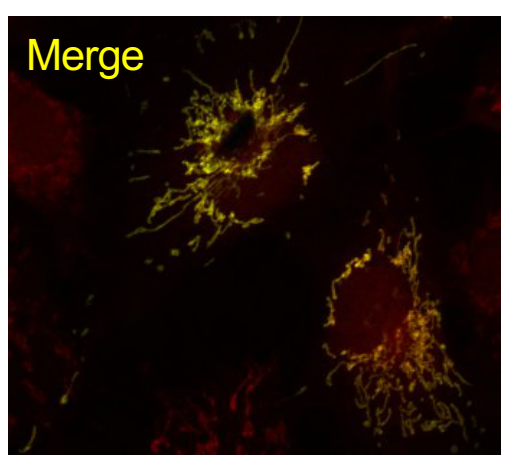

\section{Merge}

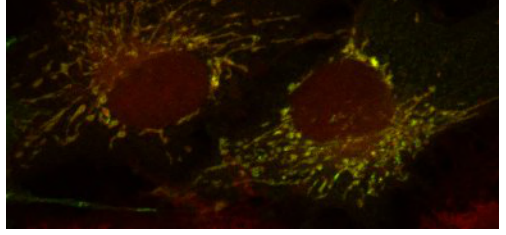

463

620

Fig 4

Mayr et al. 

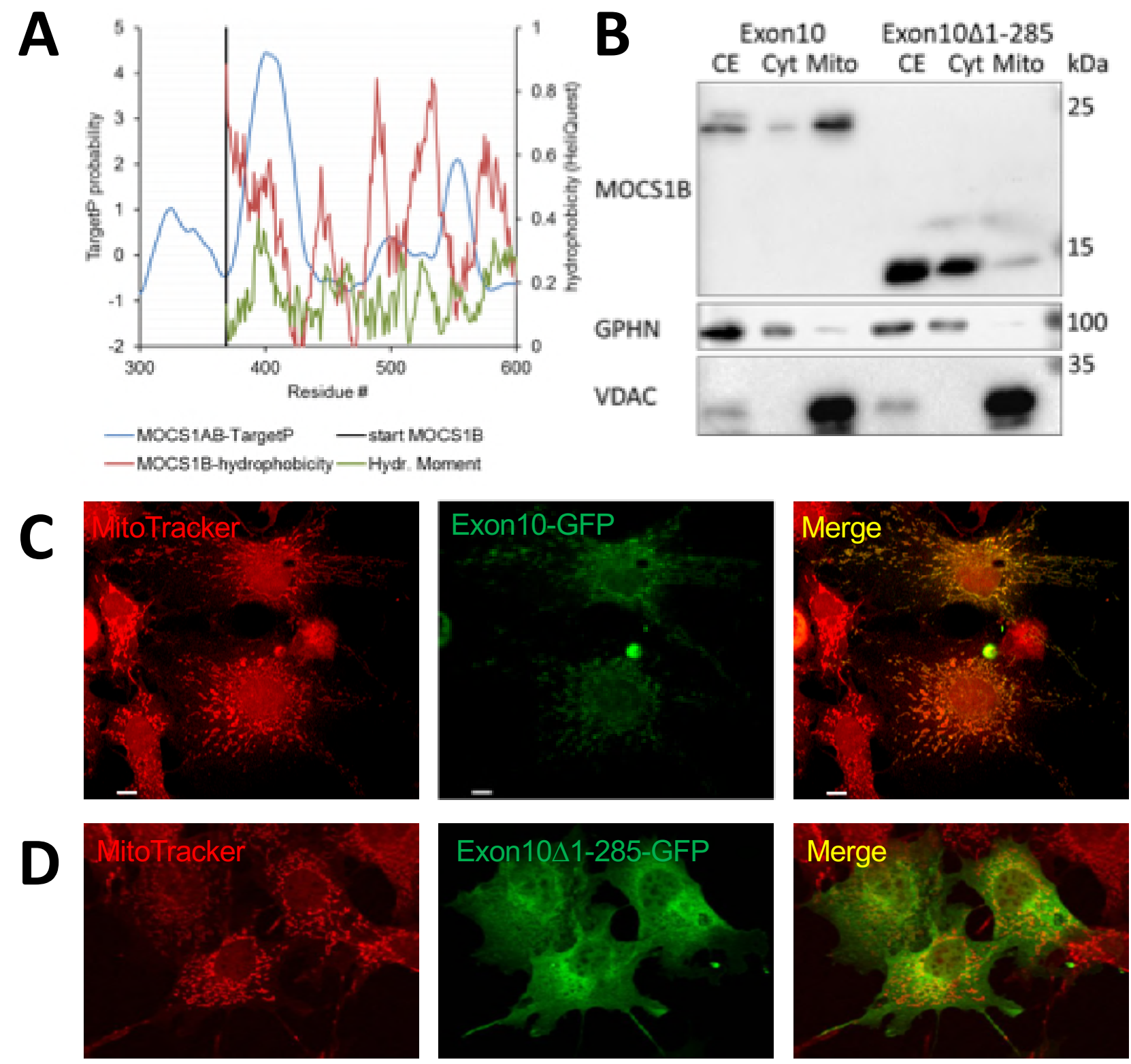

Fig 5

Mayr et al. 
A
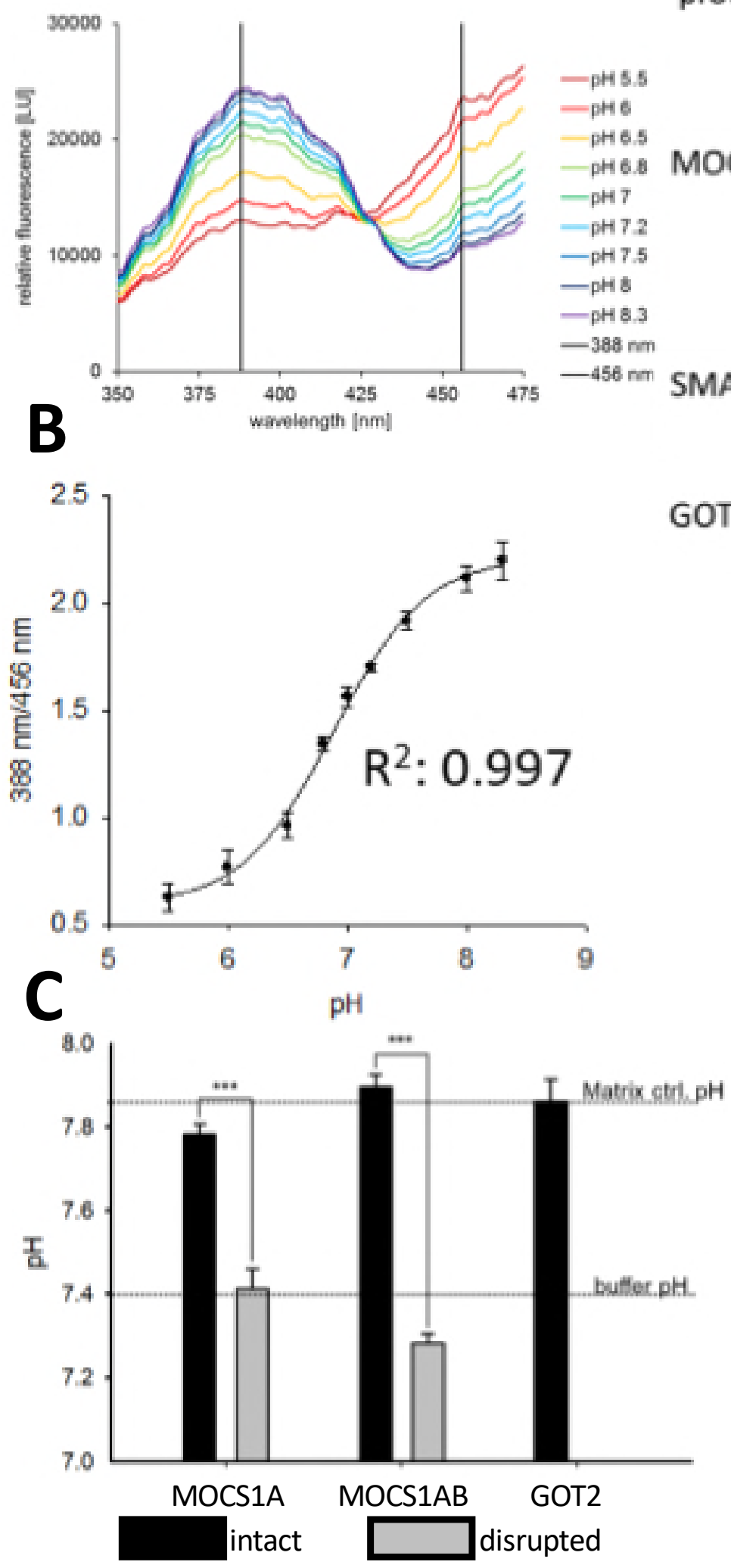

Swelling:

- $\mathrm{pH} 5.5$

- $\mathrm{pH} 6$

$\mathrm{pH} 6.5$

pH6.8 MOCS1B

$-\mathrm{pH} 7$

$-\mathrm{pH} 72$

$-\mathrm{pH} 7.5$

$-\mathrm{pH}$ है

$-\mathrm{pH} 8.3$

$-388 \mathrm{~nm}$

$-456 \mathrm{~nm}$ SMAC

GOT2

proteinasek:
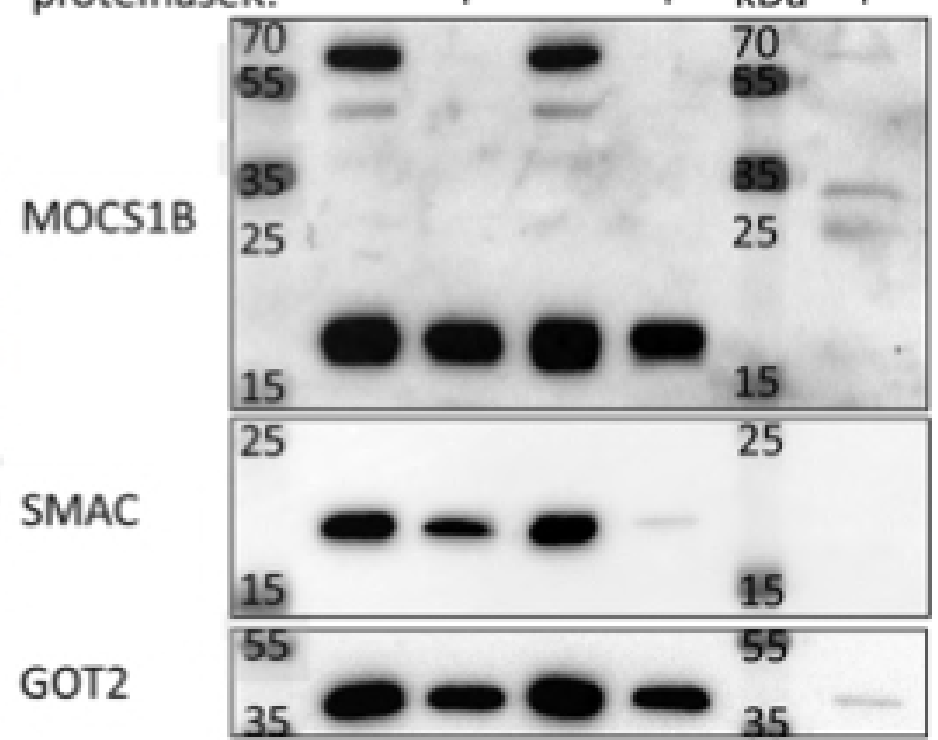

E
35-

25-

$15-$

35-

$55-$ mature

MOCS1B

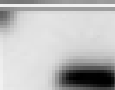

$\simeq$ VDAC

SO unprocessed MOCS1AB

Fig 6

Mayr et al. 


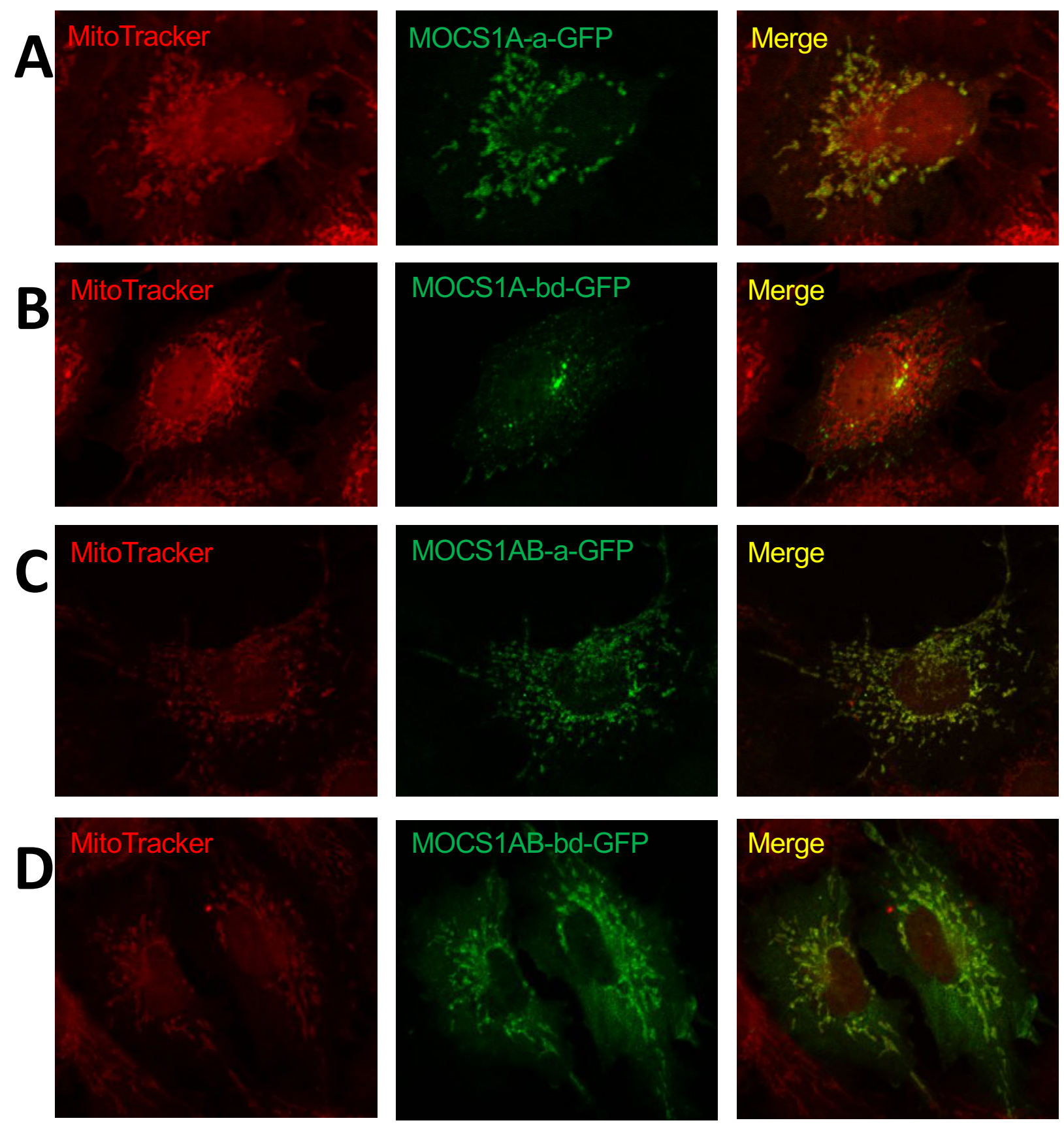

Fig EV1

Mayr et al. 

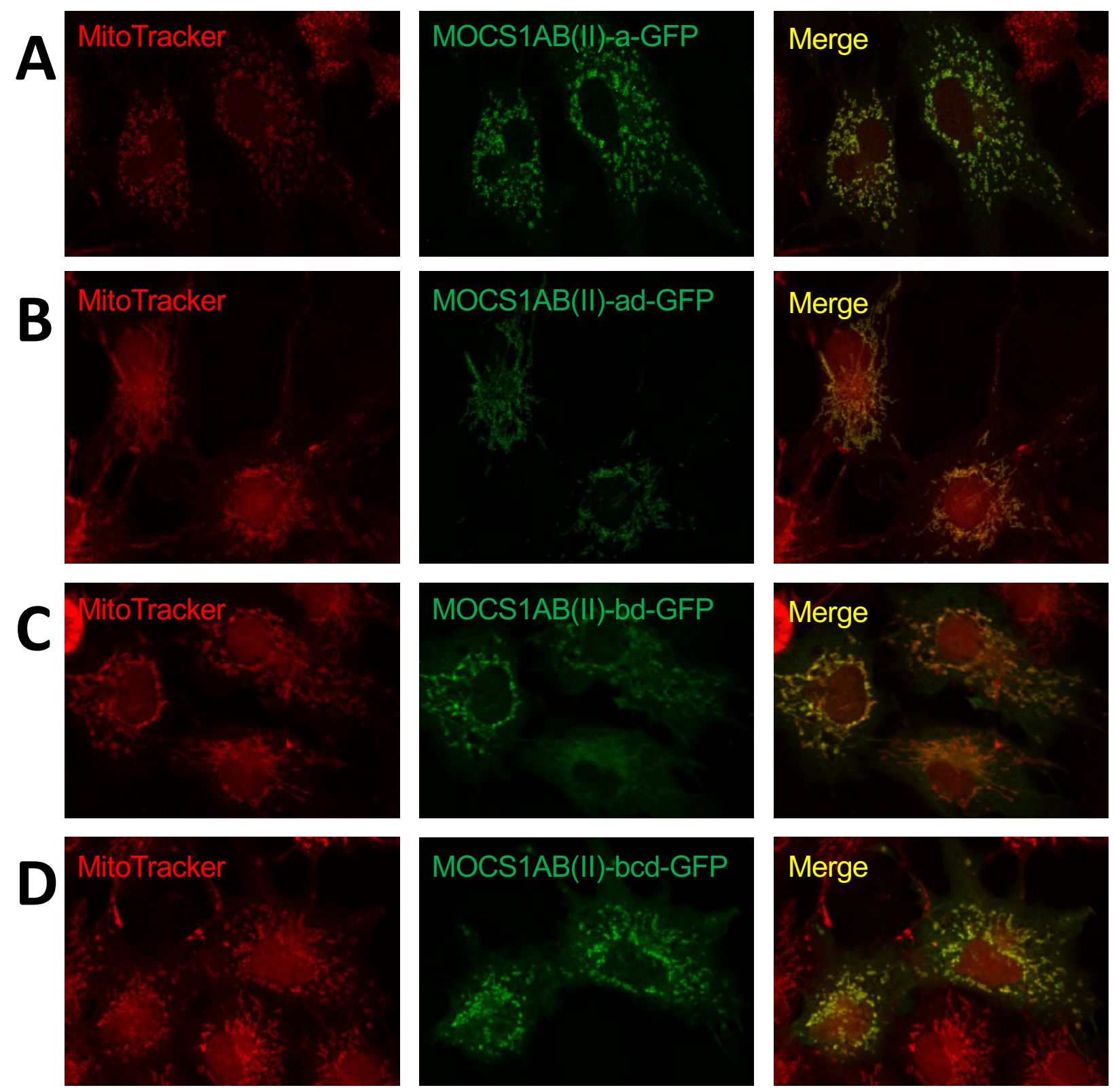

Fig EV2

Mayr et al. 

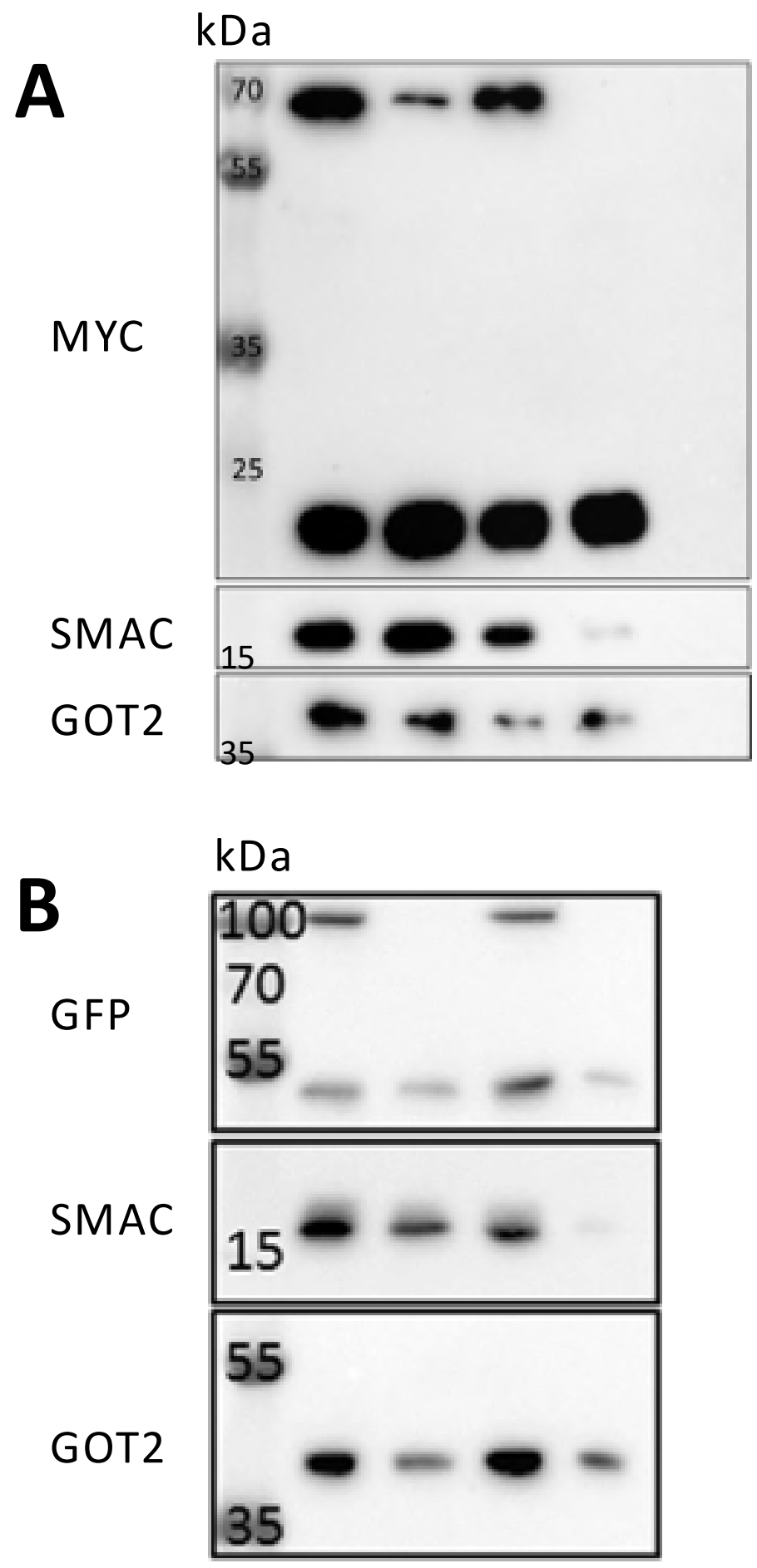

Fig EV3

Mayr et al. 


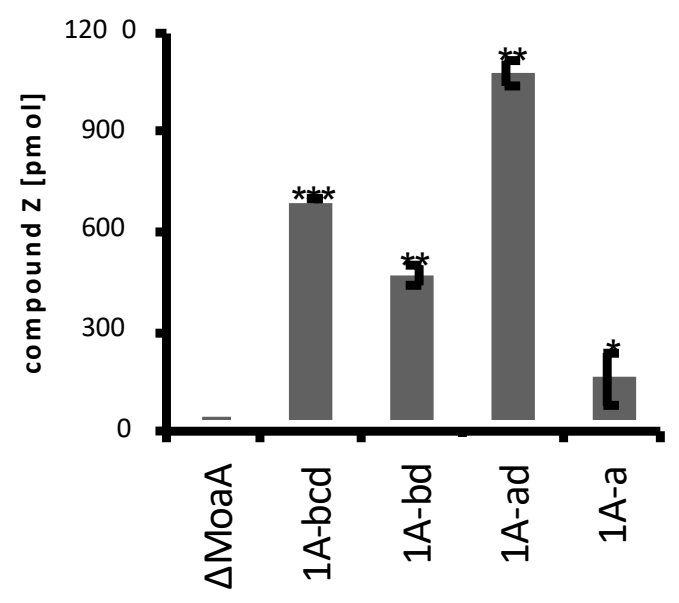

Fig EV4

Mayr et al. 
A

B

Mocs1B-antigen

HEK-Mito. $10 \mu \mathrm{g}$ Mocs1BMOCS1AB-MYC antigen
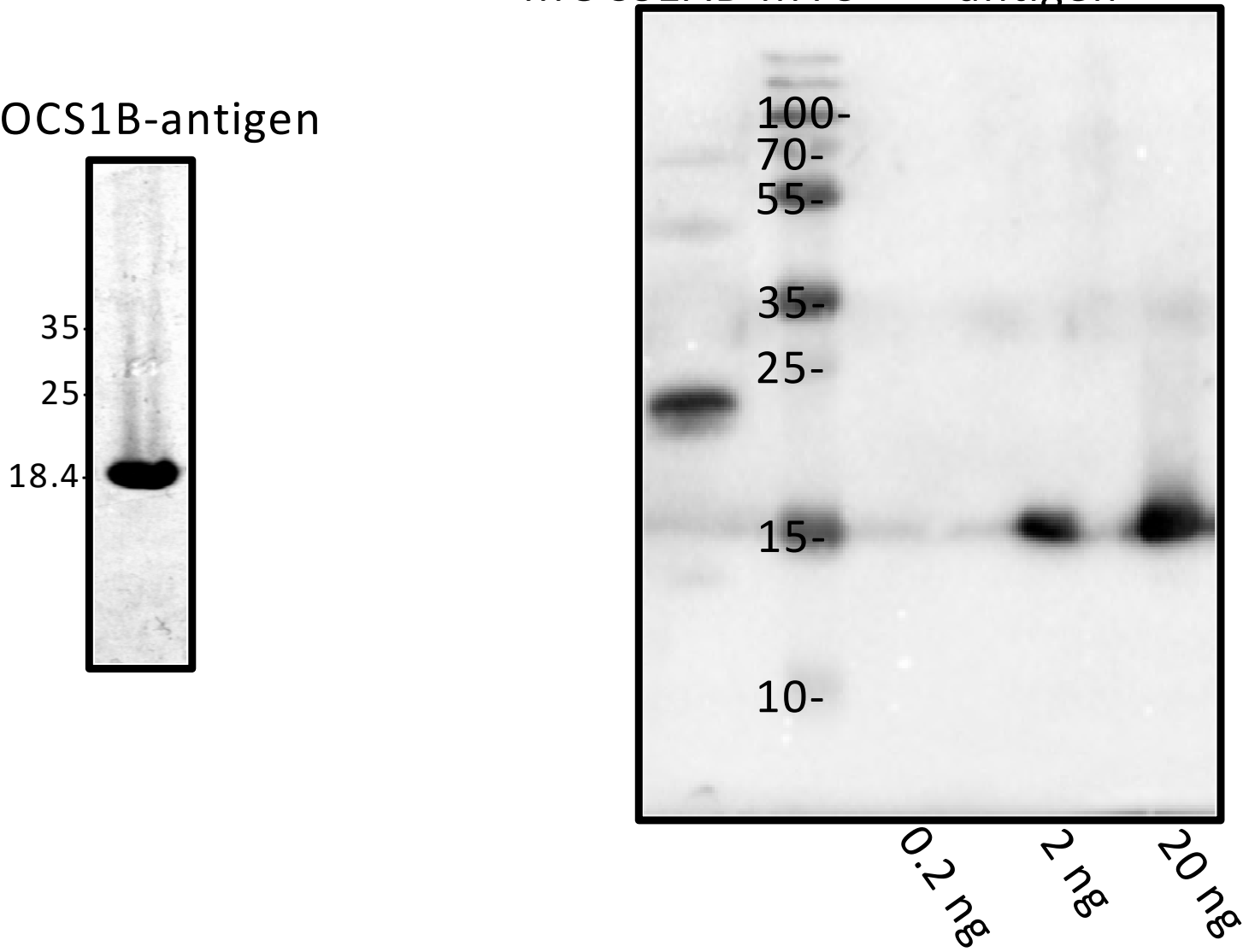

Fig EV5

Mayr et al. 